\title{
THE EULER-POISSON-DARBOUX EQUATION IN THE EXCEPTIONAL CASES ${ }^{1}$
}

\author{
E. K. BLUM
}

1. Introduction. The Cauchy problem for the Euler-PoissonDarboux (EPD) equation can be formulated as follows. Let $f\left(x_{1}, \cdots, x_{m}\right)$ be an arbitrary function satisfying certain differentiability requirements to be specified later. It is required to find a function $u\left(x_{1}, \cdots, x_{m}, t\right)$ which satisfies the three conditions

$$
\begin{aligned}
& L_{k}[u] \equiv \Delta u-u_{t t}-k t^{-1} u_{t}=0, \\
& u\left(x_{1}, \cdots, x_{m}, 0\right)=f\left(x_{1}, \cdots, x_{m}\right), \\
& u_{t}\left(x_{1}, \cdots, x_{m}, 0\right)=0,
\end{aligned}
$$

where in the EPD equation (1.1) it is understood that $\Delta u=\sum_{i=1}^{m} u_{x_{i} x_{i}}$ and $k$ is a parameter, $-\infty<k<\infty$.

We designate a solution of $L_{k}[u]=0$ by $u^{(k)}(x, t)$, writing $x$ in place of $x_{1}, \cdots, x_{m}$. While particular results for this famous equation go back to Poisson and even to Euler, a systematic investigation of the Cauchy problem covering all values of $k$ and $m$ is of recent date. The results may be summarized as follows. is

(i) If $k=m-1$, the solution, as given explicitly by Ásgeirsson [1],

$$
\begin{aligned}
& u^{(m-1)}(x, t) \\
& \quad=\frac{1}{\omega_{m}} \int_{\sum_{1}^{m} \alpha_{j}^{2}=1} \ldots \int f\left(x_{1}+\alpha_{1} t, \cdots, x_{m}+\alpha_{m} t\right) d \omega_{m},
\end{aligned}
$$

where $\omega_{m}=2(\pi)^{m / 2} / \Gamma(m / 2)$ is the area of the $m$-dimensional unit sphere.

(ii) If $k>m-1$, the solution was obtained by Weinstein [2] and is given by

$$
\begin{array}{r}
u^{(k)}(x, t)=\frac{\omega_{k+1-m}}{\omega_{k+1}} \int_{\sum_{\alpha_{j} \leqq 1}^{2}} \underset{\int}{\ln } \int f\left(x_{1}+\alpha_{1} t, \cdots, x_{m}+\alpha_{m} t\right) \\
\cdot\left(1-\sum_{1}^{m} \alpha_{j}^{2}\right)^{(k-m-1) / 2} d \alpha_{1} \cdots d \alpha_{m} .
\end{array}
$$

Received by the editors June 15, 1953.

1 This work was sponsored by the Office of Naval Research. 
(iii) For $k<m-1$ but $k \neq-1,-3,-5, \cdots$, Weinstein [3], improving on his results in [2], obtains the solution

$$
u^{(k)}(x, t)=t^{1-k}\left(\frac{\partial}{t \partial t}\right)^{n}\left[t^{k+2 n-1} u^{(k+2 n)}(x, t)\right],
$$

where $n$ is a positive integer chosen such that $k+2 n \geqq m-1$ and $u^{(k+2 n)}$ is then given by (1.4) or (1.5) with $f$ replaced by $f /(k+1)$ $\cdot(k+3) \cdots(k+2 n-1)$. Formula (1.6) generalizes the classical solution of the wave equation $(k=0)$ and provides a new and simpler means of obtaining its solution.

(iv) Another solution for $k<m-1$ is obtained by Diaz and Weinberger [4] by analytic continuation of (1.5) with respect to $k$. Their method includes the values $k=-1,-3,-5, \cdots$, the exceptional nature of which was previously discussed in [2].

It should be noted that the solution of the Cauchy problem is unique for $k>0$ whereas for $k<0$ it is not unique [2]. In particular, the solution for the exceptional values is not unique.

In this paper, we obtain by essentially different methods another solution for the exceptional cases. Our solution differs from the solution in [4] in that it is given as an explicit formula and has the further advantage that the function $f$ is required to have fewer continuous derivatives, namely, it is sufficient for $f$ to have derivatives of order at least $(m-k+3) / 2$. This generalizes a result obtained by the present author for the case of one $x$ variable $(m=1)[5 ; 7]$.

2. Two fundamental identities. Our treatment of the exceptional cases is based on the two fundamental identities

$$
\begin{aligned}
& u^{(k)}(x, t)=t^{1-k} u^{(2-k)}(x, t), \\
& u_{t}^{(k)}(x, t)=t^{-1} u^{(k+2)}(x, t),
\end{aligned}
$$

given by Darboux [6] in a slightly different form and as above in [2] and $[3]$.

We shall show that (2.1), (2.2), and (1.6) can be combined to yield a solution for $k=-1,-3,-5, \cdots$. It is convenient to rewrite these formulas, by introducing the variable $s=t^{2}$, as

$$
\begin{aligned}
u^{(k)}\left(x, s^{1 / 2}\right) & =s^{(1-k) / 2} u^{(2-k)}\left(x, s^{1 / 2}\right), \\
2 u_{s}^{(k)}\left(x, s^{1 / 2}\right) & =u^{(k+2)}\left(x, s^{1 / 2}\right), \\
u^{(k)}\left(x, s^{1 / 2}\right) & =s^{(1-k) / 2} \frac{\partial^{n}}{\partial s^{n}}\left[s^{(k+2 n-1) / 2} u^{(k+2 n)}\left(x, s^{1 / 2}\right)\right] .
\end{aligned}
$$


3. Reduction to the case $k=1$. Using (2.1a), (2.2a), we can reduce the problem for the exceptional cases to the case $k=1$. Suppose $k=-(2 r+1), r=0,1,2, \cdots$, and let $u^{(1)}(x, t)$ be a solution of $L_{1}[u]=0$. By repeated application of $(2.2 \mathrm{a})$, we have

$$
\frac{\partial^{r+1} u^{(1)}\left(x, s^{1 / 2}\right)}{\partial s^{r+1}}=u^{(2 r+3)}\left(x, s^{1 / 2}\right) .
$$

Then by (2.1a) we obtain the solution

$$
u^{-(2 r+1)}\left(x, s^{1 / 2}\right)=A s^{r+1} \frac{\partial^{r+1} u^{(1)}\left(x, s^{1 / 2}\right)}{\partial s^{r+1}},
$$

where $A$ is a constant (to be determined later) which insures that $u^{-(2 r+1)}$ assumes the prescribed initial values.

If $u^{(1)}$ is such that $\partial^{r+1} u^{(1)} / \partial s^{r+1}$ is finite for $s=0$, then clearly $u^{-(2 r+1)}(x, 0)=0$. Therefore, if (3.1) is to yield solutions for arbitrary initial values, $\partial^{r+1} u^{(1)} / \partial s^{r+1}$ should have a singularity of the type $s^{-r-1}$, that is, $u^{(1)}$ should have a singularity of the type log $s$. For $m=1$, such a solution is given in [6]. For arbitrary $m$, we construct $u^{(1)}$ as follows.

Let $k=1+2 \epsilon, \epsilon>0$. Choose the smallest positive integer $n$ such that $k+2 n>m-1$ and $2-k+2 n>m-1$. Using (1.6a) and (2.1a), we construct two solutions

$$
\begin{array}{r}
u_{1}^{(k)}\left(x, s^{1 / 2}\right) \\
=s^{(1-k) / 2} \frac{\partial^{n}}{\partial s^{n}}\left\{s^{(k+2 n-1) / 2} \int_{\sum_{1}^{m} \alpha_{j}^{2} \leqq 1} \cdots \int f\left(x_{j}+\alpha_{j} s^{1 / 2}\right)\right. \\
\left.\cdot\left(1-\sum_{1}^{m} \alpha_{j}^{2}\right)^{(k+2 n-m-1) / 2} d \alpha_{1} \cdots d \alpha_{m}\right\}, \\
u_{2}^{(k)}\left(x, s^{1 / 2}\right)=\frac{\partial^{n}}{\partial s^{n}}\left\{s^{(1-k+2 n) / 2} \int_{\sum_{1}^{m} \alpha_{j}^{2} \leqq 1} \ldots \int f\left(x_{j}+\alpha_{j} s^{1 / 2}\right)\right. \\
\left.\cdot\left(1-\sum_{1}^{m} \alpha_{j}^{2}\right)^{(1-k+2 n-m) / 2} d \alpha_{1} \cdots d \alpha_{m}\right\},
\end{array}
$$

where

$$
f\left(x_{j}+\alpha_{j} s^{1 / 2}\right) \equiv f\left(x_{1}+\alpha_{1} s^{1 / 2}, \cdots, x_{m}+\alpha_{m} s^{1 / 2}\right)
$$

and $f$ is assumed to have continuous derivatives of order at least 
$(m-k+3) / 2$. The solution $u_{2}^{(k)}$ is obtained by replacing $k$ by $(2-k)$ in (1.6a) and then applying (2.1a).

After carrying out the differentiations in (3.2) and (3.3), we have

$$
\begin{gathered}
u_{1}^{(k)}\left(x, s^{1 / 2}\right)=\sum_{q=0}^{n}\left(\begin{array}{c}
n \\
n-q
\end{array}\right) \frac{\Gamma(n+1+\epsilon)}{\Gamma(q+1+\epsilon)} s^{q} \int_{\sum_{1}^{m} \alpha_{j}^{2} \leqq 1} \ldots \frac{\partial^{q} f}{\partial s^{q}} \\
\cdot\left(1-\sum_{1}^{m} \alpha_{j}^{2}\right)^{(2 n-m+2 \epsilon) / 2} d \alpha_{1} \cdots d \alpha_{m}, \\
u_{2}^{(k)}\left(x, s^{1 / 2}\right)=\sum_{q=0}^{n}\left(\begin{array}{c}
n \\
n-q
\end{array}\right) \frac{\Gamma(n+1-\epsilon)}{\Gamma(q+1-\epsilon)} s^{q-\epsilon} \int_{\sum_{1}^{m} \alpha_{j}^{2} \leqq 1} \ldots \frac{\partial^{q} f}{\partial s^{q}} \\
\cdot\left(1-\sum_{1}^{m} \alpha_{j}^{2}\right)^{(2 n-m-2 \epsilon) / 2} d \alpha_{1} \cdots d \alpha_{m} .
\end{gathered}
$$

Now,

$$
u_{1}^{(k)}-u_{2}^{(k)}=u^{(k)}
$$

is also a solution of $L_{k}[u]=0$ and can be written as follows:

$$
\begin{gathered}
u^{(1+2 \epsilon)}\left(x, s^{1 / 2}\right) \\
=\sum_{q=0}^{n}\left(\begin{array}{c}
n \\
n-q
\end{array}\right) s^{q} \int_{\sum_{1}^{m} \alpha_{j}^{2} \leqq 1} \ldots \int \frac{\partial^{q} f}{\partial s^{q}}\left(1-\sum_{1}^{m} \alpha_{j}^{2}\right)^{(2 n-m) / 2} s^{-\epsilon / 2} \\
\cdot\left\{\frac{\left(1-\sum_{1}^{m} \alpha_{j}^{2}\right)^{\epsilon} \Gamma(n+1+\epsilon)}{\Gamma(q+1+\epsilon)} s^{\epsilon / 2}\right. \\
\left.-\frac{\left(1-\sum_{1}^{m} \alpha_{j}^{2}\right)^{-\epsilon} \Gamma(n+1-\epsilon)}{\Gamma(q+1-\epsilon)} s^{-\epsilon / 2}\right\} d \alpha_{1} \cdots d \alpha_{m} .
\end{gathered}
$$

The expression in braces is a difference for the function $\left(\left(1-\sum_{1}^{m} \alpha_{j}^{2}\right)_{j}^{\epsilon} \Gamma(n+1+\epsilon) / \Gamma(q+1+\epsilon)\right) s^{\epsilon / 2}$, formed at $\epsilon$ and $-\epsilon$. The derivative of this function at $\epsilon=0$ is

$$
C_{q}+\frac{\Gamma(n+1)}{\Gamma(q+1)} \log \left[s^{1 / 2}\left(1-\sum_{1}^{m} \alpha_{j}^{2}\right)\right],
$$

where

$$
C_{q}=\frac{d}{d \epsilon}\left[\frac{\Gamma(n+1+\epsilon)}{\Gamma(q+1+\epsilon)}\right]_{\epsilon=0} .
$$


Hence, if (3.6) is divided by $2 \epsilon$ and the limit is taken as $\epsilon$ approaches zero, a solution, $u^{(1)}$, with a logarithmic singularity is obtained formally and is given by

$$
\begin{aligned}
u^{(1)}\left(x, s^{1 / 2}\right) & =\sum_{q=0}^{n}\left(\begin{array}{c}
n \\
n-q
\end{array}\right) s^{q} \int_{\sum_{1}^{m} \alpha_{j}^{2} \leqq 1} \iint \frac{\partial^{q} f}{\partial s^{q}}\left(1-\sum_{1}^{m} \alpha_{i}^{2}\right)^{(2 n-m) / 2} \\
\cdot & \left\{C_{q}+\frac{\Gamma(n+1)}{\Gamma(q+1)} \log \left[s^{1 / 2}\left(1-\sum_{1}^{m} \alpha_{j}^{2}\right)\right]\right\} d \alpha_{1} \cdots d \alpha_{m} .
\end{aligned}
$$

To verify that $u^{(1)}$ in (3.7) is actually a solution of $L_{1}[u]=0$, we shall show that $\lim _{\epsilon \rightarrow 0} \Delta u^{(1+2 \epsilon)} / 2 \epsilon=\Delta u^{(1)}, \lim _{\epsilon \rightarrow 0} u_{s}^{(1+2 \epsilon)} / 2 \epsilon=u_{s}^{(1)}$, and $\lim _{\epsilon \rightarrow 0} u_{s s}^{(1+2 \epsilon)} / 2 \epsilon=u_{s s}^{(1)}$. Then it will follow that

$$
\lim _{\epsilon \rightarrow 0} L_{1+2 \epsilon}\left[u^{(1+2 \epsilon)} / 2 \epsilon\right]=L_{1}\left[u^{(1)}\right] .
$$

Since $L_{1+2 \epsilon}\left[u^{(1+2 \epsilon)} / 2 \epsilon\right]=0$ for $\epsilon>0$, this implies $L_{1}\left[u^{(1)}\right]=0$.

Starting from (3.6), we write $\Delta u^{(1+2 e)} / 2 \epsilon$ in the form

$$
\begin{aligned}
& \frac{\Delta u^{(1+2 \epsilon)}}{2 \epsilon}=\sum_{q=0}^{n}\left(\begin{array}{c}
n \\
n-q
\end{array}\right) s^{q} \int_{\sum_{1}^{m} \alpha_{j}^{2} \leqq 1} \ldots \int \frac{\partial^{q}(\Delta f)}{\partial s^{q}} \\
& \cdot\left(1-\sum_{1}^{m} \alpha_{j}^{2}\right)^{(2 n-m) / 2} s^{-\epsilon / 2} \\
& \left\{\frac{\left(1-\sum_{1}^{m} \alpha_{j}^{2}\right)^{\epsilon} s^{\epsilon / 2} \Gamma(n+1+\epsilon)}{2 \epsilon \Gamma(q+1+\epsilon)}-\frac{\Gamma(n+1)}{2 \epsilon \Gamma(q+1)}\right\} \\
& \cdot d \alpha_{1} \cdots d \alpha_{m} \\
& +\sum_{q=0}^{n}\left(\begin{array}{c}
n \\
n-q
\end{array}\right) s^{q} \int_{\sum_{1}^{m} \cdots} \cdots \int \frac{\partial^{q}(\Delta f)}{\partial s^{q} \leqq 1} \\
& \cdot\left(1-\sum_{1}^{m} \alpha_{j}^{2}\right)^{(2 n-m) / 2} s^{-\epsilon / 2} \\
& \left\{\frac{\frac{\Gamma(n+1)}{\Gamma(q+1)}-\left(1-\sum_{1}^{m} \alpha_{j}^{2}\right)^{-\epsilon} \frac{\Gamma(n+1-\epsilon)}{\Gamma(q+1-\epsilon)} s^{-\epsilon / 2}}{2 \epsilon}\right\} \\
& \cdot d \alpha_{1} \cdots d \alpha_{m} .
\end{aligned}
$$

From (3.7), we have 


$$
\begin{aligned}
\Delta u^{(1)}= & \sum_{q=0}^{n}\left(\begin{array}{c}
n \\
n-q
\end{array}\right) s^{q} \int_{\sum_{1}^{m} \alpha_{j}^{2} \leqq 1} \ldots \int \frac{\partial^{q}(\Delta f)}{\partial s^{q}} \\
& \cdot\left(1-\sum_{1}^{m} \alpha_{j}^{2}\right)^{(2 n-m) / 2} \\
& \cdot\left\{C_{q}+\frac{\Gamma(n+1)}{\Gamma(q+1)} \log \left[s^{1 / 2}\left(1-\sum_{1}^{m} \alpha_{j}^{2}\right)\right]\right\} d \alpha_{1} \cdots d \alpha_{m} .
\end{aligned}
$$

From the differentiability assumptions on $f$, it follows that $\partial^{q}(\Delta f) / \partial s^{q}, q=0,1, \cdots, n$, is continuous and therefore bounded in the region of integration. Furthermore, by the choice of $n$, we have $(2 n-m) / 2>-1$. Hence, for $s \neq 0$ and $\epsilon$ sufficiently small, each integrand in (3.8) is bounded by a summable function, namely,

$$
C\left(1-\sum_{1}^{m} \alpha_{j}^{2}\right)^{(2 n-m) / 2}\left\{C_{q}+\frac{\Gamma(n+1)}{\Gamma(q+1)} \log \left[s^{1 / 2}\left(1-\sum_{1}^{m} \alpha_{j}^{2}\right)\right]\right\},
$$

where $C$ is a sufficiently large constant. Thus, the Lebesgue convergence theorem is applicable and $\lim _{\epsilon \rightarrow 0} \Delta u^{(1+2 \epsilon)} / 2 \epsilon$ can be obtained by taking the limit of the integrands in (3.8). This yields precisely (3.9).

A similar procedure applies to $u_{s}^{(1+2 \epsilon)}$. We need only point out that the variable $s$ in $u^{(1)}$ and $u^{(1+2 \epsilon)}$ occurs in three places, namely, as a factor $s^{q}$, then in the derivative $\partial^{q} f / \partial s^{q}$, and finally as $s^{-\epsilon}$ in the bracketed quantity. Differentiation of the first two parts yields expressions similar to those in (3.8) and (3.9) and we can again pass to the limit as above. As for the quantity in brackets, in $u^{(1+2 \epsilon)}$, differentiation yields the expression

$$
\begin{aligned}
\sum_{q=0}^{n}\left(\begin{array}{c}
n \\
n-q
\end{array}\right) s^{q} \int_{\sum_{1}^{m} \ldots} \ldots \int \frac{\partial^{q} f}{\partial s_{j}^{2} \leqq 1}\left(1-\sum_{1}^{m} \alpha_{j}^{2}\right)^{(2 n-m) / 2} \\
\quad\left(1-\sum_{1}^{m} \alpha_{j}^{2}\right)^{-\epsilon} \frac{\Gamma(n+1-\epsilon)}{\Gamma(q+1-\epsilon)} \frac{s^{-\epsilon-1}}{2} d \alpha_{1} \cdots d \alpha_{m} .
\end{aligned}
$$

The corresponding differentiation for $u^{(1)}$ gives

$$
\sum_{q=0}^{n}\left(\begin{array}{c}
n \\
n-q
\end{array}\right) s^{q} \int_{\sum^{m} \underset{\alpha_{j}}{2} \ldots 1}^{\ldots} \int \frac{\partial^{q} f}{\partial s^{q}}
$$

$$
\cdot\left(1-\sum_{1}^{m} \alpha_{j}^{2}\right)^{(2 n-m) / 2} \frac{\Gamma(n+1)}{\Gamma(q+1)} \frac{1}{2 s} d \alpha_{1} \cdots d \alpha_{m} .
$$


It is obvious that (3.11) is the limit of (3.10) as $\epsilon$ approaches zero.

The calculations for $u_{s s}^{(1+2 \epsilon)}$ and $u_{s s}^{(1)}$ are carried out in the same way.

4. Solution for the exceptional cases. The solution, $u^{-(2 r+1)}$, of the Cauchy problem for $k=-(2 r+1)$ is given by (3.1) with $u^{(1)}\left(x, s^{1 / 2}\right)$ determined by (3.7) and the constant $A$ determined by

$$
A^{-1}=\frac{\Gamma(n+1)}{2} \int_{\sum_{1}^{m} \alpha_{j}^{2} \leqq 1} \int\left(1-\sum_{1}^{m} \alpha_{j}^{2}\right)^{(2 n-m) / 2} d \alpha_{1} \cdots d \alpha_{m} .
$$

In proof, we observe that as a consequence of the method of construction, $u^{-(2 r+1)}$ is a solution of

$$
L_{-(2 r+1)}[u]=0 .
$$

It remains to show that the initial conditions are satisfied. To do this, we shall make use of the assumption that $f$ has at least $(m-k+3) / 2$ derivatives. We recall that $n$ was chosen as the smallest integer such that $1+2 n>m-1$. Since $k=-2 r-1$, we see that our assumption means that $f$ has at least $n+r+3$ derivatives.

First, let us write the solution (3.1) in the following explicit form

$$
\begin{gathered}
u^{-(2 r+1)}\left(x, s^{1 / 2}\right) \\
=A s^{r+1} \frac{\partial^{r+1}}{\partial s^{r+1}}\left\{\sum_{q=0}^{n} C_{q}\left(\begin{array}{c}
n \\
n-q
\end{array}\right) s^{q} \int_{\sum_{1}^{m} \alpha_{j}^{2} \leqq} \ldots \int \frac{\partial^{q} f}{\partial s^{q}}\right. \\
\left.\cdot\left(1-\sum_{1}^{m} \alpha_{i}^{2}\right)^{(2 n-m) / 2} d \alpha_{1} \cdots d \alpha_{m}\right\} \\
+A s^{r+1} \frac{\partial^{r+1}}{\partial s^{r+1}}\left\{\sum_{q=0}^{n}\left(\begin{array}{c}
n \\
n-q
\end{array}\right) \frac{\Gamma(n+1)}{\Gamma(q+1)} s^{q} \int_{\sum_{1}^{m} \alpha_{j}^{2} \leqq 1} \int \frac{\partial^{q} f}{\partial s^{q}}\right. \\
\left.\cdot\left(1-\sum_{1}^{m} \alpha_{j}^{2}\right)^{(2 n-m) / 2} \log \left(1-\sum_{1}^{m} \alpha_{j}^{2}\right) d \alpha_{1} \cdots d \alpha_{m}\right\} \\
+A s^{r+1} \frac{\partial^{r+1}}{\partial s^{r+1}}\left\{\sum_{q=0}^{n}\left(\begin{array}{c}
n \\
n-q
\end{array}\right) \frac{\Gamma(n+1)}{\Gamma(q+1)} s^{q} \log s^{1 / 2}\right. \\
\left.\cdot \int_{\sum_{1}^{m} \alpha_{j}^{2} \leqq 1} \int \frac{\partial^{q} f}{\partial s^{q}}\left(1-\sum_{1}^{m} \alpha_{j}^{2}\right)^{(2 n-m) / 2} d \alpha_{1} \cdots d \alpha_{m}\right\} .
\end{gathered}
$$

Now, if we allow $s$ to approach zero, it is obvious that the first two terms in (4.2) approach zero. Carrying out the differentiation of the third term, we get for this term 


$$
\begin{aligned}
& A s^{r+1} \sum_{q=0}^{n}\left(\begin{array}{c}
n \\
n-q
\end{array}\right) \frac{\Gamma(n+1)}{2 \Gamma(q+1)} \sum_{p=1}^{r+1}\left(\begin{array}{c}
r+1 \\
p
\end{array}\right) s^{-p} \frac{\partial^{r+1-p}}{\partial s^{r+1-p}}
\end{aligned}
$$

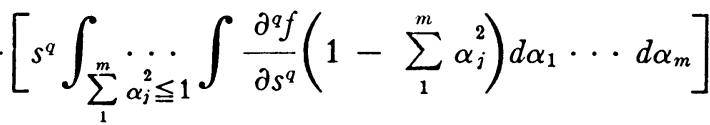

$$
\begin{aligned}
& +A(r+1) s^{r+1} \log s \sum_{q=0}^{n}\left(\begin{array}{c}
n \\
n-q
\end{array}\right) \frac{\Gamma(n+1)}{2 \Gamma(q+1)} \frac{\partial^{r+1}}{\partial s^{r+1}} \\
& \cdot\left[s^{q} \int_{\sum_{1}^{m} \underset{\alpha_{j} \leqq 1}{i} \ldots \int} \int \frac{\partial^{q} f}{\partial s^{q}}\left(1-\sum_{1}^{m} \alpha_{i}^{2}\right) d \alpha_{1} \cdots d \alpha_{m}\right] .
\end{aligned}
$$

Since $r \geqq 0$, the second term in (4.3) approaches zero as $s$ approaches zero. The first term approaches

$$
\begin{aligned}
& A \frac{\Gamma(n+1)}{2} \int_{\sum_{1}^{m}} \ldots \int \underbrace{}_{\alpha_{j} \leqq 1} \int f\left(x_{1}, \cdots, x_{m}\right)\left(1-\sum_{1}^{m} \alpha_{j}^{2}\right)^{(2 n-m) / 2} \\
& \cdot d \alpha_{1} \cdots d \alpha_{m}=f\left(x_{1}, \cdots, x_{m}\right) .
\end{aligned}
$$

A similar computation shows that $u_{t}^{-(2 r+1)}(x, 0)=0$.

5. The singular nature of the solution and polyharmonic initial values. The role of polyharmonic initial values in the exceptional cases was first pointed out in [2]. We shall see that our solution has certain properties mentioned in [2].

The singular part of $u^{-(2 r+1)}$ was written explicitly as (4.3). More precisely, the singularity is contained in the second term of (4.3). Performing the indicated differentiation, this term becomes

$$
\begin{aligned}
A(r+1) s^{r+1} & \log s \sum_{q=0}^{n} \sum_{h=0}^{r+1}\left(\begin{array}{c}
n \\
n-q
\end{array}\right) \frac{\Gamma(n+1)}{2 \Gamma(q+1)}\left(\begin{array}{c}
r+1 \\
h
\end{array}\right) \frac{d^{h} s^{q}}{d s^{h}} \\
& \int_{\sum_{1}^{m} \alpha_{j} \leqq 1} \ldots \int \frac{\partial^{p} f}{\partial s^{p}}\left(1-\sum_{1}^{m} \alpha_{j}^{2}\right)^{(2 n-m) / 2} d \alpha_{1} \cdots d \alpha_{m},
\end{aligned}
$$

where $p=r+1+q-h$. Since $d^{h} s^{q} / d s^{h}=0$ for $h>q$, we need only consider the terms for which $h \leqq q$. Each of these terms has a factor, $\Phi_{p}$, of the form

$$
\Phi_{p}=\int_{\sum_{1}^{m}} \cdots \int \frac{\partial^{p} f}{\partial s_{j}^{2} \leqq 1}\left(1-\sum_{1}^{m} \alpha_{j}^{2}\right)^{(2 n-m) / 2} d \alpha_{1} \cdots d \alpha_{m},
$$

where $p \geqq r+1$. If polar coordinates are introduced, this becomes 


$$
\Phi_{p}=\int_{0}^{1}\left(1-\rho^{2}\right)^{(2 n-m) / 2} \rho^{m-1} \int_{\sum_{1}^{m} \beta_{j}^{2}=1} \ldots \int \frac{\partial^{p} f\left(x_{j}+\beta_{j} \rho s^{1 / 2}\right)}{\partial s^{p}} d \omega_{m},
$$

where $d \omega_{m}$ is the element of area of the unit sphere in $m$-space. Now,

$$
\frac{\partial f\left(x_{i}+\beta_{j} \rho s^{1 / 2}\right)}{\partial s}=\frac{\rho}{2 s^{1 / 2}} \sum_{j=1}^{m} f_{j} \beta_{j}=\frac{\rho}{2 s^{1 / 2}} \frac{\partial f}{\partial n} .
$$

Here, $f_{j}$ denotes the partial derivative of $f$ with respect to the $j$ th variable and $\partial f / \partial n$ is the normal derivative at the point $\left(x_{1}+\beta_{1} \rho s^{1 / 2}\right.$, $\left.\cdots, x_{m}+\beta_{m} \rho s^{1 / 2}\right)$ on the surface of the sphere of radius $\rho s^{1 / 2}$. Applying the divergence theorem, we get

$$
\begin{aligned}
\Phi_{p}=\int_{0}^{1}\left(1-\rho^{2}\right)^{(2 n-m) / 2} & \frac{\rho^{m}}{2 s^{1 / 2}} \\
& \cdot\left\{\frac{\partial^{p-1}}{\partial s^{p-1}} \int_{\sum_{1}^{m} \beta_{j}^{2} \leqq 1} \int \Delta f\left(x_{j}+\beta_{j} \rho s^{1 / 2}\right) d v\right\} d \rho,
\end{aligned}
$$

where $d v$ is the volume element of the sphere of radius $\rho s^{1 / 2}$. Iterating this process $p$ times, we obtain

$$
\begin{aligned}
& \Phi_{p}=\frac{1}{\left(2 s^{1 / 2}\right)^{p}} \int_{0}^{1}\left(1-\rho^{2}\right)^{(2 n-m) / 2} \rho^{m} d \rho \int_{0}^{1} \rho_{1}^{m} d \rho_{1} \ldots \\
& \cdot \int_{0}^{1} \rho_{p-1} d \rho_{p-1} \int_{\sum_{1}^{m} \beta_{j}^{2} \leqq 1}^{\ldots} \int \Delta^{i} f\left(x_{j}+\beta_{i} \rho_{p-1} \cdots \rho_{1} \rho s^{1 / 2}\right) d v .
\end{aligned}
$$

Let us assume now that $f$ is polyharmonic of order $r+1 . \Delta^{p} f=0$ in all the factors, $\Phi_{p}$. This means that the singular term is identically zero and the solution is a polynomial in $t$, as remarked in [2]. However, if $f$ is not polyharmonic, then a straightforward differentiation of (5.1) shows that $\partial^{2 r+2} u^{-(2 r+1)} / \partial t^{2 r+2}$ becomes infinite like $\log t$ on $t=0$.

Therefore, we see that, as pointed out in [3], for the exceptional values of $k$, a small deviation, no matter how regular, from polyharmonic initial values introduces logarithmic infinities in certain derivatives of the solution.

\section{BIBLIOGRAPHY}

1. L. Åsgeirsson, Über eine Mittelwerteigenschaft von Lösungen homogener linearer partiellen Differentialgleichungen 2. Ordnung mit konstanten Koeffizienten, Math. Ann. vol. 113 (1937) pp. 321-346. (See also Courant-Hilbert, Vol. II.) 
2. A. Weinstein, Sur le problème de Cauchy pour l'équation de Poisson et l'équation des ondes, C. R. Acad. Sci. Paris vol. 234 (1952) pp. 2584-2585.

3. - On the wave equation and the equation of Euler-Poisson, Proceedings of Fif th Symposium on Applied Mathematics, McGraw-Hill, 1954.

4. J. B. Diaz and H. F. Weinberger, $A$ solution of the singular initial value problem for the Euler-Poisson-Darboux equation, Proc. Amer. Math. Soc. vol. 4 (1953) pp. 703-715.

5. E. K. Blum, A uniqueness theorem for the Euler-Poisson-Darboux equation, Bull. Amer. Math. Soc. Abstract 59-4-350.

6. G. Darboux, Lȩ̧ons sur la théorie générale des surfaces, $2 \mathrm{~d}$ ed., vol. II, Paris, 1914-15.

7. E. K. Blum, The solutions of the Euler-Poisson-Darboux equation for negative values of the parameter, Duke Math. J. (1954).

UNIVERSITY OF MARYLAND 